\title{
Exact Solutions of the Two-Dimensional Cattaneo Model Using Lie Symmetry Transformations
}

\author{
Khudija Bibi $\mathbb{D}^{1}$ and Khalil Ahmad ${ }^{2}$ \\ ${ }^{1}$ Department of Mathematics, Faculty of Basic and Applied Sciences, International Islamic University, Islamabad 44000, Pakistan \\ ${ }^{2}$ Department of Mathematics, Faculty of Basic and Applied Sciences, Air University, PAF Complex E-9, \\ Islamabad 44000, Pakistan \\ Correspondence should be addressed to Khudija Bibi; khudija.bibi@iiu.edu.pk
}

Received 17 December 2020; Revised 14 February 2021; Accepted 26 March 2021; Published 10 April 2021

Academic Editor: Paolo Renna

Copyright (c) 2021 Khudija Bibi and Khalil Ahmad. This is an open access article distributed under the Creative Commons Attribution License, which permits unrestricted use, distribution, and reproduction in any medium, provided the original work is properly cited.

\begin{abstract}
In this article, symmetry technique is utilized to obtain new exact solutions of the Cattaneo equation. The infinitesimal symmetries, linear combinations of these symmetries, and corresponding similarity variables are determined, which lead to many exact solutions of the considered equation. By applying similarity transformations, the mentioned partial differential equation is reduced to some ordinary differential equations of second order. Solutions of these ordinary differential equations have yielded many exact solutions of the Cattaneo equation.
\end{abstract}

\section{Introduction}

In the diffusion phenomenon, considered by the traditional Fick law and Fourier law, the moment a point passes by that is disturbed at a great distance, and the propagation speed of the disturbance appears infinite. However, this property is nonphysical. To handle this problem, Cattaneo proposed a model [1], in which he modified the constitutive equation by presenting a relaxation parameter which plays the role of relaxation time, where this relaxation parameter is small and depends on the thermodynamic properties of the material. From the mathematical aspect, Cattaneo model transforms the traditional diffusion equation into a hyperbolic equation, the speed of propagation is finite, and it improves the property of infinite propagation speed. On the contrary, because of the hyperbolic nature of the Cattaneo model, it can possess oscillatory solutions and negative values.

From the physical aspect, the Cattaneo model describes a physical phenomenon of heat waves. Although this phenomenon can only be observed under special states [2], circumstances, or materials, it is still gradually accepted by the community. It can be used to describe not only heat pulse propagation in some pure nonmetallic crystals [3] but also ultrasonic wave propagation in certain diluted gases. Straughan [4] considered thermal convection with the Cattaneo-Christov model in horizontal layers of an incompressible Newtonian fluid. Haddad [5] applied the theory of Cattaneo-Christov to study Brinkman's porous media. Cattaneo model is mostly used in crystalline solids [6-8], extended irreversible thermodynamics, and cosmological models.

Because of many advantages of the fractional differential equations, many scholars have introduced numerous analytical and numerical methods to study various fractional models. As Cattaneo model has wide applications in physics and theoretical analysis, so many researchers devoted themselves for the solution and generalization of the Cattaneo model. Compte and Metzler [9] generalized the Cattaneo model from three aspects which are continuoustime random walks, delayed flux-force relation, and nonlocal transport theory. Ferrillo et al. [10] compared the Cattaneo model and fractional Cattaneo model and investigated the 
asymptotic behavior of solutions to the Cattaneo equations. Su et al. [11] compared the solution of a phase-lagging heat transport equation with the solution of the classical Cattaneo equation.

There exist many well-known techniques for obtaining exact solutions of the partial differential equations [12-15], but one of them is a powerful Lie group method. By using Lie group analysis, one can also find the similarity solutions of the partial differential equations. These similarity solutions lead to the exact solutions of partial differential equations. Many researchers have used this method to find solutions of partial differential equations, e.g., see in [16-20]. In the current article, exact solutions of the Cattaneo equation via the similarity transformations are obtained.

This paper is arranged into four sections as follows: in Section 2, Lie symmetry generators of the Cattaneo equation are presented. In Section 3, the considered equation is converted into some ODEs by considering the similarity variables which are obtained by taking different linear combinations of the symmetry generators, while in Section 4, the graphical representations of solutions which are obtained in Section 3 are presented. Finally, in Section 5 , the summary of the present work is discussed.

\section{Lie Symmetries of the Cattaneo Equation}

The main goal of this paper is to investigate the exact solutions of the following Cattaneo model [21],

$$
C^{2} w_{t t}+w_{t}-D\left(w_{x x}+w_{y y}\right)=0
$$

by using its symmetry analysis. In the above model, $C$ is a relaxation parameter, and $D$ is a diffusion coefficient.

The method to obtain the Lie symmetries of the partial differential equations has been discussed in many books, e.g., see in [22-25]. Let

$$
\begin{aligned}
X= & \phi_{1}(x, y, t, w) \partial_{x}+\phi_{2}(x, y, t, w) \partial_{y}+\phi_{3}(x, y, t, w) \partial_{t} \\
& +\eta(x, y, t, w) \partial_{w}
\end{aligned}
$$

be the vector field that generates the symmetry group of equation (1). By applying the second-order prolongation of $X$ to equation (1), the following determining equations are obtained:

$$
\begin{aligned}
\eta_{t, t} & =\frac{D \eta_{y, y}+D \eta_{x, x}-\eta_{t}}{C^{2}} \\
\eta_{t, w} & =0 \\
\eta_{w, w} & =0 \\
\eta_{w, x} & =-\frac{1}{2} \frac{\left(\phi_{1}\right)_{t}}{D} \\
\left(\phi_{1}\right)_{w} & =0 \\
\left(\phi_{1}\right)_{x} & =0 \\
\left(\phi_{1}\right)_{t, t} & =0 \\
\left(\phi_{1}\right)_{t, y} & =0 \\
\left(\phi_{1}\right)_{y, y} & =0 \\
\left(\phi_{2}\right)_{w} & =0 \\
\left(\phi_{2}\right)_{x} & =-\left(\phi_{1}\right)_{y} \\
\left(\phi_{2}\right)_{y} & =0 \\
\left(\phi_{2}\right)_{t, t} & =0 \\
\left(\phi_{3}\right)_{t} & =0 \\
\left(\phi_{3}\right)_{w} & =0 \\
\left(\phi_{3}\right)_{x} & =\frac{\left(\phi_{1}\right)_{t} C^{2}}{D} \\
\left(\phi_{3}\right)_{y} & =\frac{\left(\phi_{2}\right)_{t} C^{2}}{D} \\
& \\
&
\end{aligned}
$$

The infinitesimals obtained by solving the above system are

$$
\begin{aligned}
\eta & =C_{4} w-\frac{1}{2} C_{6} \frac{w x}{D}-\frac{1}{2} C_{7} \frac{w y}{D}, \\
\phi_{1} & =C_{5} y+C_{6} t+C_{1}, \\
\phi_{2} & =-C_{5} x+C_{7} t+C_{2}, \\
\phi_{3} & =C_{6} \frac{C^{2} x}{D}+C_{7} \frac{C^{2} y}{D}+C_{3} .
\end{aligned}
$$

Thus, Cattaneo equation (1), is spanned by the following vector fields: 


$$
\begin{aligned}
& X_{1}=\partial_{x}, \\
& X_{2}=\partial_{y}, \\
& X_{3}=\partial_{t}, \\
& X_{4}=w \partial_{w} \\
& X_{5}=y \partial_{x}-x \partial_{y} \\
& X_{6}=t \partial_{x}+\frac{C^{2} x}{D} \partial_{t}-\frac{1}{2} \frac{w x}{D} \partial_{w}, \\
& X_{7}=t \partial_{y}+\frac{C^{2} y}{D} \partial_{t}-\frac{1}{2} \frac{w y}{D} \partial_{w},
\end{aligned}
$$

which form a seven-dimensional Lie algebra.

\section{Exact Solutions of the Cattaneo Model Obtained by considering Similarity Transformations}

In this section, some group-invariant solutions of Cattaneo equation (1) by considering different linear combinations of the Lie symmetry generators which have been obtained in Section 2 are presented.

(1) The similarity variables corresponding to

$$
X_{1}+X_{2}+X_{3}+X_{4}=\partial_{x}+\partial_{y}+\partial_{t}+w \partial_{w},
$$

are

$$
\begin{aligned}
v & =y-x, \\
\gamma & =t-x, \\
\mu(\nu, \gamma) & =\frac{w}{\exp (x)} .
\end{aligned}
$$

The dependent variable $\mu$ given in (7) indicates that the solution of (1) is in the form

$$
w=\mu \exp (x) .
$$

Now, we consider the independent variables given in (7) one by one to seek the solution of (1).

First, if we consider the following similarity variables,

$$
\begin{gathered}
\nu=y-x, \\
\mu(\nu)=\frac{w}{\exp (x)},
\end{gathered}
$$

then equation (1) is reduced into the following linear ODE:

$$
2 \mu_{\nu v}-2 \mu_{\nu}+\mu=0,
$$

with the solution

$$
\mu(\nu)=C_{1} \exp \left(\frac{1}{2} v\right) \sin \left(\frac{1}{2} v\right)+C_{2} \exp \left(\frac{1}{2} v\right) \cos \left(\frac{1}{2} v\right) .
$$

Now, by considering the similarity variables

$$
\begin{gathered}
\gamma=t-x, \\
\mu(\gamma)=\frac{w}{\exp (x)},
\end{gathered}
$$

equation (1) is converted into the following ODE:

$$
C^{2} \mu_{\gamma \gamma}+\mu_{\gamma}-D\left(\mu-2 \mu_{\gamma}+\mu_{\gamma \gamma}\right)=0,
$$

with the solution

$\mu(\gamma)=C_{3} \exp \left(\frac{-(1 / 2)\left(-1-2 D-\sqrt{1+4 D+4 D C^{2}}\right) \gamma}{C^{2}-D}\right)+C_{4} \exp \left(\frac{-(1 / 2)\left(-1-2 D+\sqrt{1+4 D+4 D C^{2}}\right) \gamma}{C^{2}-D}\right)$.

Since both solutions of (1) given in (11) and (14) are linearly independent,

$$
w(x, y, t)=\exp (x)(\mu(\nu)+\mu(\gamma)),
$$

i.e., 
$w(x, y, t)=\exp (x)\left(\begin{array}{c}C_{1} \exp \left(\frac{1}{2} \nu\right) \sin \left(\frac{1}{2} \nu\right)+C_{2} \exp \left(\frac{1}{2} \nu\right) \cos \left(\frac{1}{2} \nu\right)+C_{3} \exp \left(\frac{-(1 / 2)\left(-1-2 D-\sqrt{1+4 D+4 D C^{2}}\right) \gamma}{C^{2}-D}\right)+ \\ C_{4} \exp \left(\frac{-(1 / 2)\left(-1-2 D+\sqrt{1+4 D+4 D C^{2}}\right) \gamma}{C^{2}-D}\right)\end{array}\right)$.

Putting values of $v$ and $\gamma$ in the above equation, we obtain that

$$
w(x, y, t)=\exp (x)\left(\begin{array}{c}
C_{1} \exp \left(\frac{1}{2}(y-x)\right) \sin \left(\frac{1}{2}(y-x)\right)+C_{2} \exp \left(\frac{1}{2}(y-x)\right) \cos \left(\frac{1}{2}(y-x)\right) \\
+C_{3} \exp \left(\frac{-(1 / 2)\left(-1-2 D-\sqrt{1+4 D+4 D C^{2}}\right)(t-x)}{C^{2}-D}\right)+ \\
C_{4} \exp \left(\frac{-(1 / 2)\left(-1-2 D+\sqrt{1+4 D+4 D C^{2}}\right)(t-x)}{C^{2}-D}\right)
\end{array}\right),
$$

(2) The similarity variables corresponding to

$$
X_{5}=y \partial_{x}-x \partial_{y}
$$

are

$$
\begin{gathered}
\nu=t, \\
\gamma=x^{2}+y^{2}, \\
\mu(\nu, \gamma)=w .
\end{gathered}
$$

The dependent variable $\mu$ given in (19) indicates that the solution of (1) is in the form

$$
w=\mu \text {. }
$$

Again, we consider the independent variables one by one given in (19) to seek solutions of (1). Thus, by using $v=t$ and $\mu(v)=w$, equation (1) is transformed into an ODE

$$
\mu_{\nu}+C^{2} \mu_{\nu v}=0
$$

with the solution

$$
\mu(\nu)=C_{1} \exp \left(\frac{-v}{C^{2}}\right)+C_{2} .
$$

And by considering equation (1) is transformed into the following ODE:

$$
\mu_{\gamma}+\gamma \mu_{\gamma \gamma}=0
$$

with the solution

$$
\mu(\gamma)=C_{3} \ln (\gamma)+C_{4} .
$$

By combining solutions given in (22) and (25), we get that

$w(x, y, t)=C_{1} \exp \left(\frac{-\nu}{C^{2}}\right)+C_{3} \ln (\gamma)+C_{2}+C_{4}$,

i.e.,

$w(x, y, t)=C_{1} \exp \left(\frac{-t}{C^{2}}\right)+C_{3} \ln \left(x^{2}+y^{2}\right)+C_{5}$,

is a solution of (1).

(3) The similarity variables corresponding to

$$
X_{4}+X_{5}=y \partial_{x}-x \partial_{y}+\partial_{w}
$$




$$
\begin{aligned}
v & =x^{2}+y^{2}, \\
\gamma & =t \\
\mu(\nu, \gamma) & =\frac{w}{\exp (\arctan (x / y))} .
\end{aligned}
$$

The dependent variable $\mu$ given in (29) indicates that the solution of (1) is in the form

$$
w=\mu \exp \left(\arctan \left(\frac{x}{y}\right)\right)
$$

By considering

$$
\begin{array}{r}
v=x^{2}+y^{2}, \\
w(\nu)=\frac{w}{\exp (\arctan (x / y))},
\end{array}
$$

equation (1) is transformed into the following ODE:

$$
v^{2} \mu_{\nu v}+v \mu_{v}+\frac{1}{4} \mu=0
$$

with the solution

$$
\mu(\nu)=C_{1} \sin \left(\frac{1}{2} \ln (\nu)\right)+C_{2} \cos \left(\frac{1}{2} \ln (\nu)\right) .
$$

Thus,

$$
w(x, y, t)=\exp \left(\arctan \left(\frac{x}{y}\right)\right) \mu(\nu) \text { or } w(x, y, t)=\exp \left(\arctan \left(\frac{x}{y}\right)\right)\left(C_{1} \sin \left(\frac{1}{2} \ln (\nu)\right)+C_{2} \cos \left(\frac{1}{2} \ln (\nu)\right)\right)
$$

i.e.,

$$
w(x, y, t)=\exp \left(\arctan \left(\frac{x}{y}\right)\right)\left(C_{1} \sin \left(\frac{1}{2} \ln \left(x^{2}+y^{2}\right)\right)+C_{2} \cos \left(\frac{1}{2} \ln \left(x^{2}+y^{2}\right)\right)\right)
$$

is a solution of (1).

Here, we neglect the similarity variables $\gamma=t$ and $\mu(\gamma)=(w / \exp (\arctan (x / y))) \quad$ because corresponding to these variables, equation (1) is not transformed into an ODE.

(4) The similarity variables corresponding to

$$
X_{6}=t \partial_{x}+\frac{C^{2} x}{t} \partial_{t}-\frac{1}{2} \frac{w x}{D} \partial_{w}
$$

are

$$
\begin{aligned}
\nu & =y \\
\gamma & =\frac{-C^{2} x^{2}+t^{2} D}{D}, \\
\mu(\nu, \gamma) & =\frac{w}{\exp \left(-(1 / 2)\left(t / C^{2}\right)\right)} .
\end{aligned}
$$

By considering $v=y \quad$ and $\mu(\nu)=\left(w / \exp \left(-(1 / 2)\left(t / C^{2}\right)\right)\right)$, equation (1) is transformed into an ODE

$$
D \mu_{\nu v}+\frac{1}{4 C^{2}} \mu=0
$$

with the solution

$$
\mu(\nu)=C_{1} \sin \left(\frac{1}{2} \frac{\nu}{C \sqrt{D}}\right)+C_{2} \cos \left(\frac{1}{2} \frac{v}{C \sqrt{D}}\right)
$$

Now, by considering

$$
\begin{gathered}
\gamma=\frac{-C^{2} x^{2}+t^{2} D}{D}, \\
\mu(\gamma)=\frac{w}{\exp \left(-(1 / 2)\left(t / C^{2}\right)\right)},
\end{gathered}
$$

equation (1) is transformed into the following ODE:

$$
16 C^{4} \gamma \mu_{\gamma \gamma}+16 C^{4} \mu_{\gamma}-\mu=0 \text {, }
$$

with the solution

$$
\mu(\gamma)=C_{3} I\left(\frac{1}{2} \frac{\sqrt{\gamma}}{C^{2}}\right)+C_{4} K\left(\frac{1}{2} \frac{\sqrt{\gamma}}{C^{2}}\right),
$$

where $I\left((1 / 2)\left(\sqrt{\gamma} / C^{2}\right)\right)$ and $K\left((1 / 2)\left(\sqrt{\gamma} / C^{2}\right)\right)$ are the modified Bessel functions of the first and second 


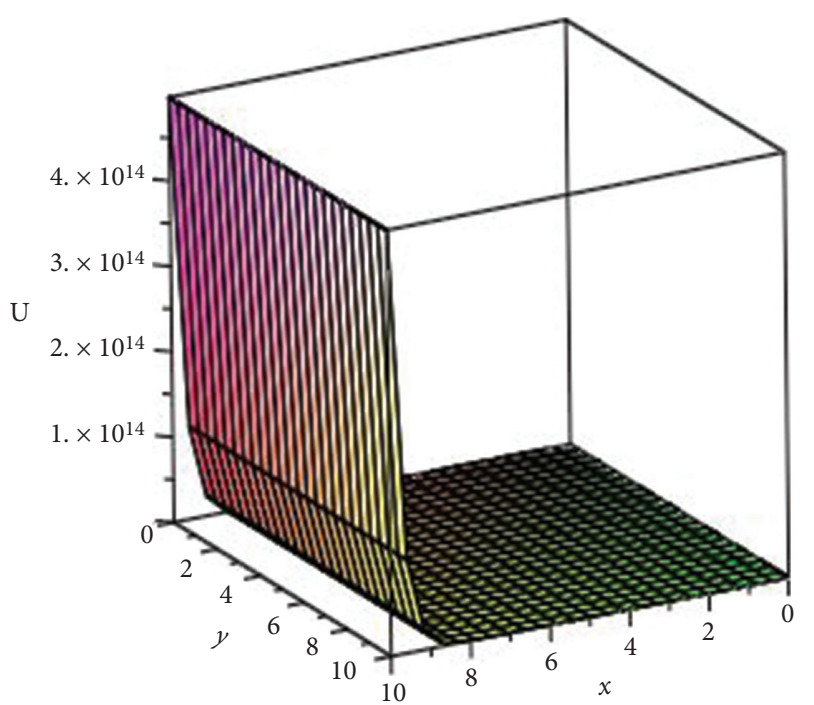

Figure 1: Graph of $w$ given by (17) for $C=0.1, D=1$, and $C_{1}=C_{2}=C_{3}=C_{4}=1$.

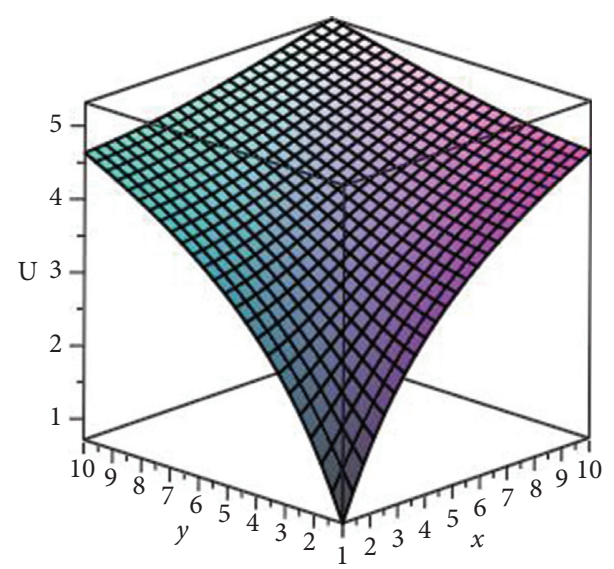

Figure 2: Graph of $w$ given by (27) for $C=0.5, C_{1}=C_{3}=1$, and $C_{5}=0$.

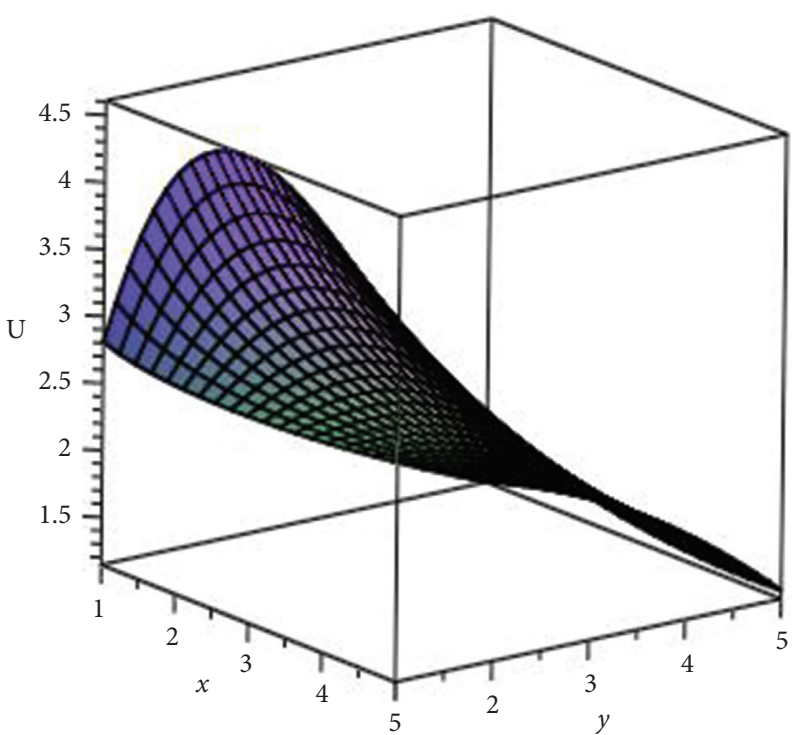

Figure 3: Graph of $w$ given by (35) for $C_{1}=C_{2}=1$. 


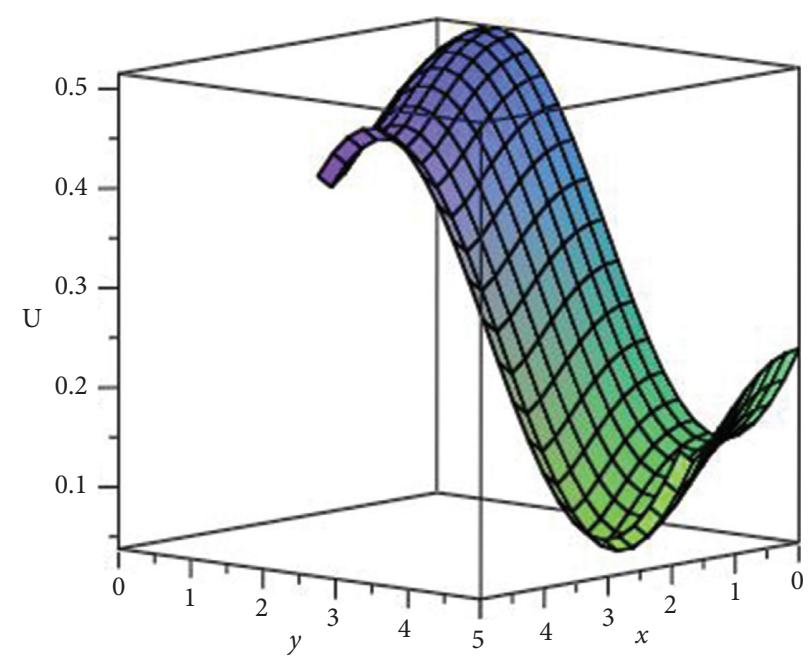

Figure 4: Graph of $w$ given by (44) for $C=0.5, D=1$, and $C_{1}=C_{2}=C_{3}=C_{4}=1$.

kinds, respectively. By combining (39) and (42), we

have

$$
\begin{aligned}
& w(x, y, t)=\exp \left(-\frac{1}{2} \frac{t}{C^{2}}\right)(\mu(\nu)+\mu(\gamma)) \\
& w(x, y, t)=\exp \left(-\frac{1}{2} \frac{t}{C^{2}}\right)\left(C_{1} \sin \left(\frac{1}{2} \frac{\gamma}{C \sqrt{D}}\right)+C_{2} \cos \left(\frac{1}{2} \frac{\nu}{C \sqrt{D}}\right)+C_{3} I\left(\frac{1}{2} \frac{\sqrt{\gamma}}{C^{2}}\right)+C_{4} K\left(\frac{1}{2} \frac{\sqrt{\gamma}}{C^{2}}\right)\right)
\end{aligned}
$$

i.e.,

$$
w(x, y, t)=\exp \left(-\frac{1}{2} \frac{t}{C^{2}}\right)\left(C_{1} \sin \left(\frac{1}{2} \frac{y}{C \sqrt{D}}\right)+C_{2} \cos \left(\frac{1}{2} \frac{y}{C \sqrt{D}}\right)+C_{3} I\left(\frac{1}{2} \frac{\sqrt{\left(-C^{2} x^{2}+t^{2} D / D\right)}}{C^{2}}\right)+C_{4} K\left(\frac{1}{2} \frac{\sqrt{\left(-C^{2} x^{2}+t^{2} D / D\right)}}{C^{2}}\right)\right)
$$

is a solution of (1).

\section{Surface Graphs of the Obtained Solutions}

In this section, the surface graphs of the exact solutions of the Cattaneo model which are calculated in the previous section are presented. Figures 1-4 show the surface graphs of the exact solutions of the considered model given in equations (17), (27), (35), and (44), respectively.

\section{Summary and Conclusions}

In this paper, the authors have applied the Lie symmetry method to the Cattaneo equation for obtaining its exact solutions. On achieving the similarity variables, equation (1) is reduced to some ODEs of second order. Finally, the authors have obtained some solutions of undergone ODEs including Bessel functions, which lead to many exact solutions of the considered equation. The surface graphs of solutions are presented to show different behaviors of the considered model. This paper has an interesting application of the Lie group method in a manner that a PDE with three independent variables is directly converted into an ODE by considering its different Lie symmetry generators.

\footnotetext{
Abbreviations

$\mu: \quad$ A similarity dependent variable which is a function of two independent variables

$v$ : A similarity independent variable

$\gamma$ : A similarity independent variable

$C_{1}, C_{2}, C_{3}, C_{4}$, Constants of integration

$C_{5}$ :

$I$ and $K$ : $\quad I$ and $K$ presented in equations (42) and (44) are the modified Bessel functions of the first and second kinds, respectively.
} 


\section{Data Availability}

No data were used to support this study.

\section{Conflicts of Interest}

The authors declare that they have no conflicts of interest.

\section{References}

[1] C. Cattaneo, "Sulla conduzione del calore," Atti del Seminario Matematico e Fisico dell'Universit di Modena, vol. 3, pp. 83101, 1948.

[2] B. Straughan, Heat Waves, Springer Science Business, New York, NY, USA, 2011.

[3] K. V. Zhukovsky and H. M. Srivastava, "Analytical solutions for heat diffusion beyond Fourier law," Applied Mathematics and Computation, vol. 293, pp. 423-437, 2017.

[4] B. Straughan, "Thermal convection with the Cattaneo christov model," International Journal of Heat and Mass Transfer, vol. 53, no. 1-3, pp. 95-98, 2010.

[5] S. A. M. Haddad, "Thermal instability in Brinkman porous media with Cattaneo-Christov heat flux," International Journal of Heat and Mass Transfer, vol. 68, no. 4, pp. 659-668, 2014.

[6] D. Jou, J. Casas-Vazquez, and G. Lebon, "Extended irreversible thermodynamics," Reports on Progress in Physics, vol. 51, no. 8, pp. 1105-1179, 1988.

[7] M. Zakari and D. Jou, "Equations of state and transport equations in viscous cosmological models," Physical Review D, vol. 48, no. 4, pp. 1597-1601, 1993.

[8] S. Godoy and L. S. García-Colín, "From the quantum random walk to classical mesoscopic diffusion in crystalline solids," Physical Review E, vol. 53, no. 6, pp. 5779-5785, 1996.

[9] A. Compte and R. Metzler, "The generalized Cattaneo equation for the description of anomalous transport processes," Journal of Physics A: Mathematical and General, vol. 30, no. 21, pp. 7277-7289, 1997.

[10] F. Ferrillo, R. Spigler, and M. Concezzi, "Comparing Cattaneo and fractional derivative models for heat transfer processes," SIAM Journal on Applied Mathematics, vol. 78, no. 3, pp. 1450-1469, 2018.

[11] S. Su, W. Dai, P. M. Jordan, and R. E. Mickens, "Comparison of the solutions of a phase-lagging heat transport equation and damped wave equation," International Journal of Heat and Mass Transfer, vol. 48, no. 11, pp. 2233-2241, 2005.

[12] A. Yusuf, "Symmetry analysis, invariant subspace and conservation laws of the equation for fluid flow in porous media," International Journal of Geometric Methods in Modern Physics, vol. 17, no. 12, 2020.

[13] M. Alquran, I. Jaradat, and A. Yusuf, "Heart-cusp and bellshaped-cusp optical solitons for an extended two-mode version of the complex Hirota model: application in optics," Optical and Quantum Electronics, vol. 53, no. 26, 2021.

[14] A. Yusuf, T. Abdulkadir Sulaiman, E. M. Khalil, M. Bayram, and H. Ahmad, "Construction of multi-wave complexiton solutions of the Kadomtsev-Petviashvili equation via two efficient analyzing techniques," Results in Physics, vol. 21, 2021.

[15] M. Alquran, F. Yousef, F. Alquran, T. A. Sulaiman, and A. Yusuf, "Dual-wave solutions for the quadratic-cubic conformable-Caputo time-fractional Klein-Fock-Gordon equation," Mathematics and Computers in Simulation, vol. 185, pp. 62-76, 2021.
[16] K. Sakkaravarthi, A. G. Johnpillai, A. Durga Devi, T. Kanna, and M. Lakshmanan, "Lie symmetry analysis and group invariant solutions of the nonlinear Helmholtz equation," Applied Mathematics and Computation, vol. 331, p. 457, 2018.

[17] C. Li and J. Zhang, "Lie symmetry analysis and exact solutions of generalized fractional zakharov-kuznetsov equations," Symmetry, vol. 11, no. 5, 2019.

[18] H. Liu, J. Li, and Q. Zhang, "Lie symmetry analysis and exact explicit solutions for general Burgers' equation," Journal of Computational and Applied Mathematics, vol. 228, no. 1, p. 19, 2009.

[19] P. J. Olver, Applications of Lie Groups to Differential Equations, Springer-Verlag, New York, NY, USA, 1986.

[20] K. Bibi and K. Ahmad, "Exact solutions of newell whiteheadsegel equations using symmetry transformations," Journal of Function Spaces, vol. 2021, Article ID 6658081, 8 pages, 2021.

[21] Y. Huang and Z. Yin, "The compact finite difference method of two-dimensional Cattaneo model," Journal of Function Spaces, vol. 2020, 12 pages, 2020.

[22] N. H. Ibragimov, CRC Handbook of Lie Group Analysis of Differential Equations, CRC Press, Boca Raton, FL, USA, 1996.

[23] H. Stephani, Differential Equations: Their Solutions Using Symmetries, Cambridge University Press, Cambridge, UK, 1989.

[24] G. W. Bluman and S. Kumei, Symmetries and Differential Equations, Springer-Verlag, New York, NY, USA, 1989.

[25] P. J. Olver, Equivalnce, Invariants and Symmetry, Cambridge University Press, Cambridge, UK, 1995. 